\title{
Pertinent clinical outcomes in pediatric survivors of pediatric acute respiratory distress syndrome (PARDS): a narrative review
}

\author{
Siew Wah Lee ${ }^{1,2}$, Sin Wee Loh ${ }^{1}$, Chengsi Ong ${ }^{3,4}$, Jan Hau Lee ${ }^{1,5}$ \\ ${ }^{1}$ Children's Intensive Care Unit, KK Women's and Children's Hospital, Singapore; ${ }^{2}$ Pediatric Intensive Care Unit, Kuala Lumpur Hospital, Kuala \\ Lumpur, Malaysia; ${ }^{3}$ Department of Nutrition and Dietetics, KK Women's and Children's Hospital, Singapore; Biochemistry, Yong Loo Lin School \\ of Medicine, National University of Singapore, Singapore; ${ }^{5}$ Duke-NUS Medical School, Singapore \\ Contributions: (I) Conception and design: SW Lee, JH Lee; (II) Administrative support: None; (III) Provision of study materials or patients: None; (IV) \\ Collection and assembly of data: SW Lee, JH Lee; (V) Data analysis and interpretation: SW Lee, JH Lee; (VI) Manuscript writing: All authors; (VII) \\ Final approval of manuscript: All authors. \\ Correspondence to: Jan Hau Lee. Children's Intensive Care Unit, KK Women's and Children's Hospital, 100 Bukit Timah Road, 229899 Singapore, \\ Singapore. Email: lee.jan.hau@singhealth.com.sg.
}

\begin{abstract}
The objectives of this review are to describe the limitations of commonly used clinical outcomes [e.g., mortality, ventilation parameters, need for extracorporeal membrane oxygenation (ECMO), pediatric intensive care unit (PICU) and hospital length of stay (LOS)] in pediatric acute respiratory distress syndrome (PARDS) studies; and to explore other pertinent clinical outcomes that pediatric critical care practitioners should consider in future clinical practice and research studies. These include long-term pulmonary function, risk of pulmonary hypertension (PHT), nutrition status and growth, PICU-acquired weakness, neurological outcomes and neurocognitive development, functional status, health-related quality of life (HRQOL)], health-care costs, caregiver and family stress. PubMed was searched using the following keywords or medical subject headings (MESH): "acute lung injury (ALI)", "acute respiratory distress syndrome (ARDS)", "pediatric acute respiratory distress syndrome (PARDS)", "acute hypoxemia respiratory failure", "outcomes", "pediatric intensive care unit (PICU)", "lung function”, "pulmonary hypertension”, "growth”, “nutrition', "steroid", "PICU-acquired weakness", "functional status scale", "neurocognitive", "psychology", "healthcare expenditure", and "HRQOL". The concept of contemporary measure outcomes was adapted from adult ARDS long-term outcome studies. Articles were initially searched from existing PARDS articles pool. If the relevant measure outcomes were not found, where appropriate, we considered studies from nonARDS patients within the PICU in whom these outcomes were studied. Long-term outcomes in survivors of PARDS were not follow-up in majority of pediatric studies regardless of whether the new or old definitions of ARDS in children were used. Relevant studies were scarce, and the number of participants was small. As such, available studies were not able to provide conclusive answers to most of our clinical queries. There remains a paucity of data on contemporary clinical outcomes in PARDS studies. In addition to the current commonly used outcomes, clinical researchers and investigators should consider examining these contemporary outcome measures in PARDS studies in the future.
\end{abstract}

Keywords: Acute lung injury; acute respiratory distress syndrome (ARDS); pediatric acute respiratory distress syndrome (PARDS); acute hypoxemia respiratory failure; outcomes; pediatric intensive care unit (PICU)

Submitted Mar 12, 2019. Accepted for publication Aug 26, 2019.

doi: 10.21037/atm.2019.09.32

View this article at: http://dx.doi.org/10.21037/atm.2019.09.32 


\section{Introduction}

Pediatric acute respiratory distress syndrome (PARDS) is an uncommon diagnosis in the pediatric intensive care unit (PICU), with a reported incidence of only 3\% (1). Despite its low incidence, mortality from PARDS remains high. A systematic review reported a pooled PARDS mortality rate of $24 \%$ [ $95 \%$ confidence interval (CI): $19-31 \%$ ], which has decreased over time from $40 \%$ before 2000 (95\% CI: $24-59 \%)$ to $18 \%$ (95\% CI: $12-26 \%)$ after 2010 (2). The overall reduction in mortality rate has been attributed to earlier recognition of the disease, advances in critical care, use of early lung protective strategies and improvement in supportive care (3). Unfortunately, approximately a quarter of patients developed new morbidities after PARDS (4). These morbidities may be the result of residual organ dysfunctions from PARDS, complications from treatments, or a combination of both (4). Understanding the consequences of PARDS is important so that clinicians can modify provision of care to minimize the morbidity risks, ensure quality improvement initiatives within the PICU, focused on these important outcomes, and formulate a better management plan to reduce hospital and PICU readmissions. Figure 1 summarizes the events that lead to PARDS and subsequent potential outcomes.

Many adult survivors of acute respiratory distress syndrome (ARDS) experience impairment in cognition, mental health, and physical function known as postintensive care syndrome (PICS) $(5,6)$. ARDS survivors demonstrate persistent physical and psychosocial problems up to 5 years post ARDS (7). These impairments potentially limit their ability to return to work and can constitute a significant burden on their families and on the society (7). Similarly, pediatric survivors of PARDS may face similar issues. The post intensive care syndrome in pediatrics (PICS-p) framework highlights the importance of baseline status which will influence the eventual trajectory of recovery, organ system maturation, psychosocial development, the interdependence of family, and trajectories of health recovery that can potentially impact a child's life for decades (8).

Conventional clinical outcomes, such as mortality, ventilation parameters, need for extracorporeal membrane oxygenation (ECMO) and PICU or hospital length of stay (LOS), which are often used in clinical studies, may not be the most appropriate endpoints for contemporary PARDS clinical trials. Recent report from the Pediatric Acute Lung Consensus Conference (PALICC) proposed that PARDS may pose significant personal, social and economic burden, suggesting the need to explore other relevant outcome measures that include patient- (and caregiver-) reported outcomes, long-term developmental milestones in a child, as well as economic cost analysis (9). These include long-term pulmonary function, risk of pulmonary hypertension (PHT), nutrition status and growth, PICU-acquired weakness, neurological outcomes and neurocognitive development, functional status, health-related quality of life (HRQOL), health-care costs, caregiver and family stress. Unfortunately, there is currently a paucity of data on these contemporary outcome measures (9).

Thus, the objectives of this narrative review are to describe the limitations of currently used clinical outcomes in published PARDS studies; to explore new outcomes that pediatric critical care practitioners should consider in clinical practice and research studies; to depict the current utility of patient-reported outcomes in PARDS and finally to characterize future approaches that can be considered in clinical studies in PARDS when describing these pertinent outcomes.

\section{Methods}

PubMed was searched using the following keywords or medical subject headings (MESH) terms in various combinations: "acute lung injury (ALI)", "acute respiratory distress syndrome (ARDS)", "pediatric acute respiratory distress syndrome (PARDS)", "acute hypoxemia respiratory failure", "outcomes", "pediatric intensive care unit", "lung function", "pulmonary hypertension", "growth", "nutrition', "steroid", "PICU-acquired weakness", "functional status scale", "neurocognitive", "psychology", "health-care expenditure", and "HRQOL". The concept of contemporary measure outcomes was adapted from adult ARDS long-term outcome studies $(5,10)$. Articles were initially searched from existing PARDS articles pool. If the relevant measure outcomes were not found, where appropriate, we considered studies from non-ARDS patients within the PICU in whom these outcomes were studied.

\section{Results}

\section{Conventional outcome measures}

\section{Mortality}

Outcomes such as PICU mortality, 28-day mortality, 60-day mortality are easily obtainable short-term outcome measures which are frequently used in clinical trials. In 


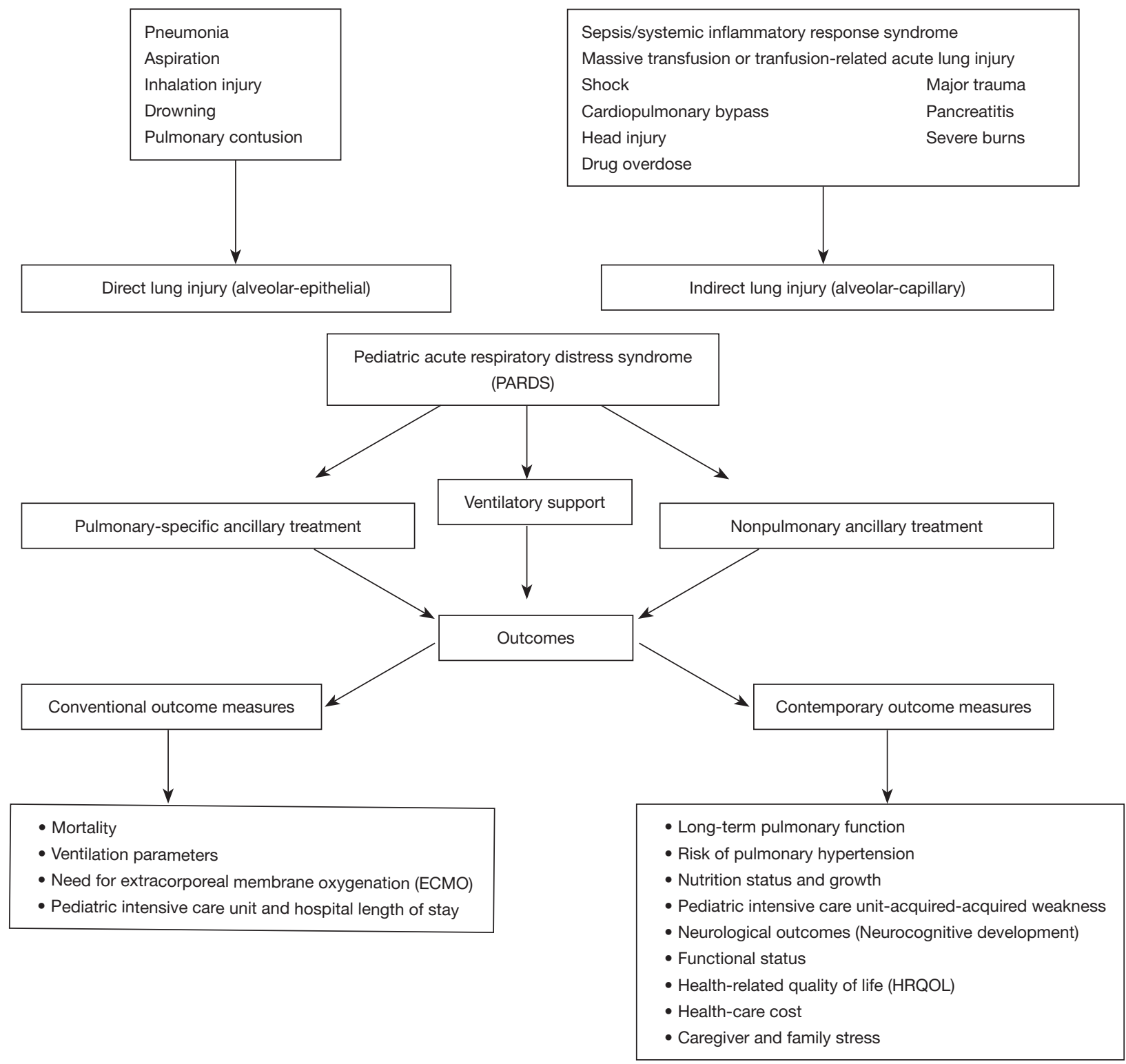

Figure 1 Overview of pediatric acute respiratory distress syndrome (PARDS).

contrast to in-hospital mortality which is usually affected by disease severity, comorbidities and the use of life-supporting technologies $(11,12)$, longer-term mortality may be a better indicator on how well the health-care providers maintain the wellness of PARDS survivors. Improved survival has greatly increased the number of patients needed to achieve statistical significance to demonstrate reduction in mortality, hence making recruitment difficult for clinical trials. It is estimated that 1,500-2,500 adult patients are now needed to achieve significance in mortality rate for phase 3 clinical trials, as compared to 860 patients recruited for the ARDS
Network trial (3). Given the low mortality in children with PARDS, future PARDS trials would similarly require large numbers of patients.

It has become increasingly apparent that existing definitions of ARDS include a highly heterogenous group of patients with considerable different comorbidities and severity. Many have questioned the usage of mortality as a short-term clinical outcome for ARDS as patients with ARDS usually die from organ failure associated with underlying comorbidities (e.g., immunocompromised status) or complications from disease progression (e.g. 
multiorgan failure and sepsis) rather than respiratory failure itself (13-16). It is postulated that attributing mortality to PARDS might be an over generalization in many PARDS deaths as PARDS has often resolved at the time of death (16).

\section{Ventilation parameters}

Duration of mechanical ventilation (MV) and ventilation free days (VFD) are common outcomes used in PARDS clinical trials (17-19). VFD at 28 days is derived from subtracting duration of MV from 28 days. Both outcomes are limited to survivors, negating the potential effects of interventions on non-survivors in the clinical trials. The use of ventilation parameters as outcome measures in clinical trials will also be increasingly challenged by the shift of focus from treating established ARDS to preventing ARDS, eventually leading to decreasing need for invasive MV (20). Furthermore, definition of ventilation parameters might differ between clinical trials depending on whether the trial includes non-invasive ventilation as part of the management, which has been recognized as part of the definition of ARDS since 2012 (21). In addition, duration of $\mathrm{MV}$ can be lengthened by poor secretions control, poor respiratory effort from neurological comorbidities or airway abnormalities. Patients may have recovered from PARDS but remained intubated for other reasons, limiting the use of ventilation parameters as a short-term outcome in PARDS clinical trials.

\section{Need for ECMO}

Need for ECMO as an outcome measure is based on the premise that the degree of hypoxemia in PARDS is so severe that it is refractory to "safe" conventional MV support. This outcome measure is not applicable in non-ECMO centers even though some might argue that it is equivalent to mortality in such instances (15). Furthermore, ECMO should be considered more of an intervention rather than an outcome and is highly dependent on hospital resources and physicians' threshold (18). The indications and contraindications also differ among different centers, making it a challenge to consider this an outcome in multicenter clinical trials.

\section{PICU and hospital LOS}

Clinical trials that used PICU and hospital LOS as outcome measures often find no significant difference in LOS between intervention and placebo group $(19,22)$. It is a commonly used outcome measure as it is easy to obtain and patient-centered. However, patients who recovered from
PARDS might stay in PICU or hospital for reasons such as caregiver training, rehabilitation and lack of caregivers, making LOS a poor outcome measurement of PARDS. Similar to other clinical conditions, LOS is also significantly affected by non-clinical factors and adjustment for these factors is often necessary $(17,23)$.

\section{Contemporary outcome measures to consider}

\section{Long-term pulmonary function}

There are limited studies that report on the long-term pulmonary function in survivors of PARDS. Available studies are often of small sample size and are not limited to children with PARDS alone.

One of such studies which consisted of 29 children (age 5-12 years old) who required MV for acute hypoxemic respiratory failure showed a significant proportion of patients had subclinical pulmonary function abnormalities within the first year of follow-up (24). These patients were follow-up at 3 and 9-12 months after discharge from PICU. During the first visit, 38\% (11/29) of patients had recurrent respiratory symptoms, $14 \%$ (4/29) of patients had abnormal chest radiographs, and $83 \%$ (24/29) of patients had abnormal spirometry readings. During the second visit, none had recurrent respiratory symptoms and only $19 \%(5 / 27)$ still had abnormal spirometry readings. This cohort showed a significant improvement in spirometry test over few months $(\mathrm{P}<0.001)$. For the spirometry test, majority demonstrated a restrictive $[71 \%(17 / 24)$ vs. $60 \%$ $(3 / 5)]$ picture at both visits (24). This finding was different from another study that found none of the patients exhibited restrictive lung disease (25). In this particular study, 24\% (4/17) had obstructive lung disease, 12\% (2/17) had abnormal diffusing capacity of the lungs for carbon monoxide (DLCO) and the rest had normal pulmonary function tests at nearest to the 12-month post ARDS (25).

Another smaller study which recruited 7 children with ARDS (mean duration of $M V$ was $9.4 \pm 7.3$ days and worst $\mathrm{PaO}_{2} / \mathrm{FiO}_{2}$ ratio was $65.1 \pm 17.0 \mathrm{mmHg}$ ) and they were followed-up for a mean duration of $5.6 \pm 4.3$ years after PICU discharge. Pulmonary functions were within normal limits in all patients except for one child who had mildly reduced DLCO and another who had mild exercise-induced hypoxemia. None of them were symptomatic or had physical limitations (26).

It appears that majority PARDS survivors had no prolonged lung sequelae in all these studies. However, given the small sample size, future clinical studies should include 
the presence of respiratory symptoms, oxygen dependence, physical limitations, exacerbations, and lung function abnormalities in PARDS survivors.

\section{Risk of PHT}

Studies on risk of PHT in children with PARDS are also lacking. Most studies reported early development of PHT in ARDS; however, incidence of late PHT in ARDS survivors remains poorly described (27-31). Using trans-esophageal echocardiographic (TEE) at day 3 of $\mathrm{MV}$, a longitudinal study of adult ARDS patients reported the incidence of acute cor pulmonale to be $25 \%(19 / 75)$; and they had complete resolution of PHT by the end of 4 weeks (29). In children, it was reported as high as $21 \%$ of patients had PHT early in their course of PARDS (28).

Mechanisms of PHT in these patients include endothelial dysfunction, pulmonary vascular occlusion, increased vascular tone, extrinsic vessel occlusion, and vascular remodeling (30-32). Pathogenesis of elevated pulmonary vascular resistance and PHT may be related to the interaction between alveolar over-distension and blood flow in patients that are ventilated with high airway pressure $(31,33)$.

Patients with cardiac and respiratory disease often display depressed exercise capacity (34-36). The functional walk tests [e.g., six-minute walk test (6MWT)] (35) and the cardiopulmonary exercise test (CPET) (36) with metabolic monitoring are among the modalities to assess exercise capacity (37). A study which followed up 29 children (mean age $8.4 \pm 2.4$ years) who received $M V$ for acute hypoxemic respiratory failure at $3.5 \pm 1.2$ and $10.6 \pm 2.7$ months after discharge from PICU showed the mean distance walked increased significantly from $352 \pm 66.7$ to $401 \pm 60.7$ minutes, $\mathrm{P}=0.002$ (24). However, these children were not tested formally for evidence of PHT in this study. Therefore, the causes of depressed exercise capacity in this group of patients remain unknown.

Extrapolating from patients with congenital heart disease, electrocardiograph (ECG) can potentially detect PHT before they develop right heart failure symptoms (38). The ECG can provide suggestive evidence of PHT by demonstrating right ventricular hypertrophy (39). However, the sensitivity is low despite a good specificity ( $>95 \%)$ and a positive predictive value of $12 \%$ in an adult study $(40,41)$. Thus, ECG alone may be inadequate as a screening tool to pick up PHT in children with PARDS. Invasive cardiac catheterization remains the gold standard for detection of elevated pulmonary pressures (42). However, this is often not practical in pediatric survivors of PARDS.
Therefore, echocardiography remains the most common screening modality for PHT as it is noninvasive and readily available (43). There remains limited description of the degree and natural history of PHT in PARDS. Because PHT potentially impacts the exercise capability of survivors, future studies should consider reporting PHT as one of the short-term outcome measures in PARDS.

\section{Nutrition status and growth}

Children have poor body reserves and are dependent on adults for care-giving. Therefore, they are at risk of malnutrition. There are numerous barriers to ensure adequate enteral nutrition (EN) in critically ill children, including feed interruptions, intolerance and fluid restrictions (44). The benefits of EN have been demonstrated in numerous studies (45-48). However, both underfeeding and overfeeding are associated with worse outcomes (49).

Adult survivors of critical illness demonstrate skeletal muscle wasting as evidenced by reduced rectus femoris cross-sectional (CSA) through serial ultrasound measurements with associated functional impairment and was severe among those with multiorgan failure (50). Muscle mass is maintained through balanced protein breakdown and synthesis (51). In PARDS, whether muscle wasting occurs, and whether this is persistent is currently unknown, but deserves further study. Therefore, continued monitoring of nutritional status remains paramount even after discharge.

Whilst there are data supporting the association between high admission body mass index and lower mortality risk in PARDS, the impact of PARDS and current clinical management on post-PICU growth remain largely unknown (52). Somatic growth and biological maturation are influenced by several factors that act independently and in concert to modify a child's genetic growth potential. Some of the management strategies in PARDS may have consequences on growth such as the use of corticosteroids $(53,54)$. In an animal study, early postnatal exposure to steroid has been shown to cause significant growth deficits in weanling and adolescent rats (55). Similar findings were also found in preterm babies treated with steroids postnatally for their bronchopulmonary dysplasia (BPD) (56). These infants had decreased absolute growth velocities during and shortly after treatment, followed by an increase in growth velocity thereafter (57). Other significant findings in the prior-mentioned animal study were changes in metabolic hormones, thus raising concerns for early onset 
of adult diseases such as obesity, diabetes, hypertension, and cardiovascular disease (55). Given this preliminary data in animal studies, investigators should consider following-up growth parameters in PARDS survivors, especially those who has been treated with corticosteroids.

\section{PICU-acquired weakness}

ICU-acquired weakness (i.e., critical illness myopathy and critical illness neuropathy) occurs in a significant proportion of critically ill adults $(50,57)$. However, a large national database reported a much lower incidence of PICU-acquired weakness in critically ill children $[0.03 \%$ $(55 / 203,875)]$ (58). This low incidence might be due under-recognition and under-reporting due to no standard diagnostic criteria for ICU-acquired weakness in children. The importance of recognizing and reporting the incidence of PICU-acquired weakness is that duration of $M V$ was higher among children with PICU-acquired weakness versus those who did not develop PICU-acquired weakness $(31.6 \pm 28.9$ vs. $9.3 \pm 20.6 ; \mathrm{P}<0.001)(58)$. Therapies utilized in PARDS such as neuromuscular blockade, corticosteroids can potentially contribute to PICU-acquired weakness. In addition to that, this outcome should be followed-up in PARDS survivors as it potentially impacts subsequent recovery, as demonstrated in a study that examined school attendance of 29 children (5-12 years old) who were ventilated for acute hypoxemic respiratory failure. School attendance was only $55.2 \%(16 / 29)$ at first followup $(3.5 \pm 1.2$ months $)$ and $85.2 \%(23 / 27)$ at the second visit (10.6 \pm 2.7 months) $(\mathrm{P}<0.001)$. One main reason for not attending school was perception of weakness among parents and patients (seven patients during first follow-up) (24). However, it was not specifically described in the study whether the investigators documented associated PICUacquired weakness in these patients.

\section{Neurological outcomes (neurocognitive development)}

Appropriate neurocognitive development can determine a child's ability to be self-sufficient, independent, sociable and functioning in the society. However, certain therapies used in PARDS may potentially impact on neurological outcomes in survivors.

In animals, there was evidence to suggest that hydrocortisone had long lasting negative effect toward neurodevelopment, learning abilities and memory outcomes that persisted into adulthood, with more marked effects in those treated with higher steroid dose (55). However, the negative impacts of steroid on cognitive functions were not replicated in a randomized clinical trial of extremely preterm infants treated with early low-dose hydrocortisone to prevent BPD. There was no significant difference in neurodevelopment at 2 years of age (59).

Steroid effects on the brain mimic an inverse-Ushaped curve, because deleterious effects result from both glucocorticoid insufficiency and/or excess glucocorticoid tissue exposure (60). Taking into consideration that preterm infants are at higher risks of neurodevelopment disabilities, extrapolation of these findings to PARDS survivors may be inappropriate. Indeed, further studies are required to determine the effects of corticosteroids on PARDS survivors.

\section{Functional status}

Functional status, defined as the ability to carry out everyday activities, is increasingly recognized as an important outcome measure of the critical illness and its treatment-related complications (61). The functional status scale (FSS) was developed by a multidisciplinary consensus process to facilitate assessment of functional status in large PICU outcome studies. The FSS, a clinical score across 6 domains of function on a 1 to 5 scale, is applicable for patients across all age groups and developmental stages, and is fast and easy to use (61). New impairments as measured by the FSS appear to be an intermediate outcome for PICU patients on the spectrum between survival with perfect health and death, reinforcing the relevance of functional impairment as an outcome measure (62). One study utilized the FSS to determine new morbidity rate of PARDS survivors (4). New morbidity, defined as an increase (worsening) in FSS of at least 3 points from baseline (pre-illness) at hospital discharge, was present in $23 \%(63 / 274)$ of PARDS survivors (4). The greatest degree of impairment occurred in motor function, respiratory and feeding domains. However, as the FSS is a measure of global function, it is unable to capture the functional impairment in greater detail (e.g., the degree of lung capacity reduction, etc.).

In addition to FSS, there are many other existing functional outcome measures available for use in pediatrics. These include Adaptive Behavioral Assessment System (ABAS-III), Vineland Adaptive Behavioral Scales (Vineland $\left.^{\mathrm{TM}}-\mathrm{II}\right)$, Bayley Scales of Infant and Toddler Development (Bayley-III), pediatric cerebral performance category (PCPC) and pediatric overall performance category (POPC). ABAS-III, Vineland ${ }^{\mathrm{TM}}$-II, and Bayley-III provide complete assessment of age-appropriate functional skills but can be time-consuming to perform. The PCPC 
and POPC were developed to quantify short-term cognitive impairment and overall short-term functional morbidity, respectively (63). PCPC and POPC are easy and quick to perform but may involve subjectivity and future projections by the assessors (61). The ideal tool for measurement of functional status outcomes in PICU or PARDS survivors remains unclear and requires further study and standardization.

\section{HRQOL}

Advances in intensive care have changed what were acute life-threatening events previously to chronic illness with protracted course. It is imperative that the healthcare team is aware of the long-term impact of PARDS survivorship (64). Children with chronic health conditions are at increased risk of HRQOL problems compared to their healthy peers $(65)$. In a small pilot study $(n=24)$ on HRQOL in PARDS survivors, children reported worse perceptions of their general health compared to children with chronic asthma (25). By learning the most affected domains of HRQOL in PARDS survivors, the healthcare team can start to address these areas so as to improve the HRQOL of these survivors. Thus, HRQOL is an important outcome measure in both clinical practice and research areas in PARDS.

The importance of HRQOL in ARDS was demonstrated from adult studies. A prospective cohort study of 200 adult patients was conducted to measure the quality-adjusted survival in the first year after ARDS. Using the quality of well-being $(\mathrm{QWB})$ scale $(0$ to 1 scale where $1=$ optimal well-being) measured at 6 and 12 months, investigators determined the quality-adjusted life-years (QALYs) of the survivors. The mean QWB was reported to be low with a narrow confidence interval $(0.59 \pm 0.015$ and $0.60 \pm 0.015$ at 6 and 12 months, respectively, $\mathrm{P}=0.48)$. This represented a considerable illness burden, and probably occurred as a direct result of their critical illness (66).

\section{Health-care costs}

Health care costs associated with PARDS should also be considered as a pertinent clinical outcome. Critical illness can add significant financial strain for patients and their families. This includes daily financial burdens, work absenteeism, and impaired productivity for parents caring for a child with a PICU admission (67). The PICU admission-related daily expenses could sometimes exceed their estimated ideal daily budgets and it was more often occurred in lower-income groups than in higher-income groups (67). Sadly, in some countries, parents had to forgo the medical treatment for their children due to financial difficulties (68).

In addition to medical expenses, there are significant non-medical out-of-pocket expenses (NMOOPEs) as well. A prospective single-center study, administering a daily verbal response survey on NMOOPEs, stratified families by annual income ( $<$ USD \$50,999, \$51-99,000, >\$100,000); and calculated daily expenditures (DEs), estimated daily budgets (DBs), and percentage of NMOOPEs (\%DE/DB). The study included 38 families, with median length of PICU stay of 3 days (range, 3-13 days). Financial impact of DB in the 3 annual income groups were $0 \%$ to $136 \%$ (median $36 \%$ ), $5 \%$ to $18 \%$ (median $10 \%$ ), and $4 \%$ to $39 \%$ (median 16\%), respectively (67), suggesting a considerable non-medical cost regardless of income group.

Even though majority [72.3\% (198/274)] of PARDS survivors were discharged home, a small percentage of them require admission to a care facility for various reasons [24.5\% (67/274)] to a rehabilitation facility; 3.3\% (9/274) to a long-term chronic care facility (4). Those who require subsequent readmissions can still impose significant cost to the total health-care expenditures. For example, in the United States, median cost per patient can range from USD $\$ 42,181$ in the low-cost hospitals to USD\$82,588 in the high-cost hospitals (69). The inclusion of health-care costs in the study gave us an idea how to manage the hospital resources but the relevant data was not always easy to be collected.

Till date, there remain a limited number of studies that examined the cost-effectiveness of the various therapies for PARDS. Future studies examining therapies of PARDS should consider including a cost-effectiveness in their overall analyses.

\section{Caregiver and family stress}

Family routine is often disrupted whenever a child is admitted to the PICU. Emotional, physical and financial stresses are common among parents and caregivers. The reason for this could be due to changes in the appearance and condition of a critically ill child, parental role alteration, procedures, sights and sounds within the PICU (70). A study involving 118 parents of 91 children ( 74 mothers and 44 fathers) was conducted to assess the psychological outcomes in parents of critically ill hospitalized children using the Speilberger State-Trait Anxiety Inventory- 
state anxiety subscale (STAI), Center for Epidemiological Studies-Depression (CES-D) and Decisional Conflict Scale (DCS). This study showed that $24 \%$ of parents had severe anxiety (STAI $>60$ ), $51 \%$ had possibility of major depression (CES-D >21) and 26\% displayed significant conflict (DCS >37.5) (71). Another prospective study was done to examine the long-term effects of the PICU experience on parents and on family adaptation. Data were obtained from 63 parents from the 2 groups of patients [parents with children in the PICU vs. parents with children in a general care unit (GCU)]. Mothers whose child was in the PICU had significantly higher Parental Stressor Scale: PICU (PSS: PICU) total scores compared to those in the GCU. Some families were still in the dysfunctional range even 6 months after discharge. Another study also reported a high levels of distraction in working families and also interrupted or suspended normal daily activities (70). Hence, this is another important component to be considered in future PARDS studies.

\section{Conclusions}

Although currently available studies that examine and report long-term outcomes in survivors of PARDS are limited, we expect this number to increase over the next few years. Clinicians and researchers can adapt experience from other pediatric chronic lung diseases or adult ARDS studies to study these contemporary outcomes. The advantages and disadvantages of each conventional and contemporary measure outcomes are summarized in Tables 1 and 2. As a medical community, we should start moving away from only describing the traditional clinical outcomes such as duration of MV, PICU stay and mortality. Future studies in PARDS are required to study the impact of PARDS on subsequent

Table 1 Conventional clinical outcomes

\begin{tabular}{lll}
\hline Conventional outcome measures & Advantages & Disadvantages \\
\hline Mortality & Convenient & $\begin{array}{l}\text { Need a greater number of patients to achieve statistical significance } \\
\text { given the low mortality in PARDS }\end{array}$ \\
& & The death may not be due to PARDS \\
Ventilation parameters & Convenient & Limited to survivors \\
Need for ECMO & Convenient & Not applicable in non-ECMO centers \\
PICU and hospital length of stay & Convenient & Potentially confounded by other factors \\
\hline
\end{tabular}

Table 2 Contemporary clinical outcomes

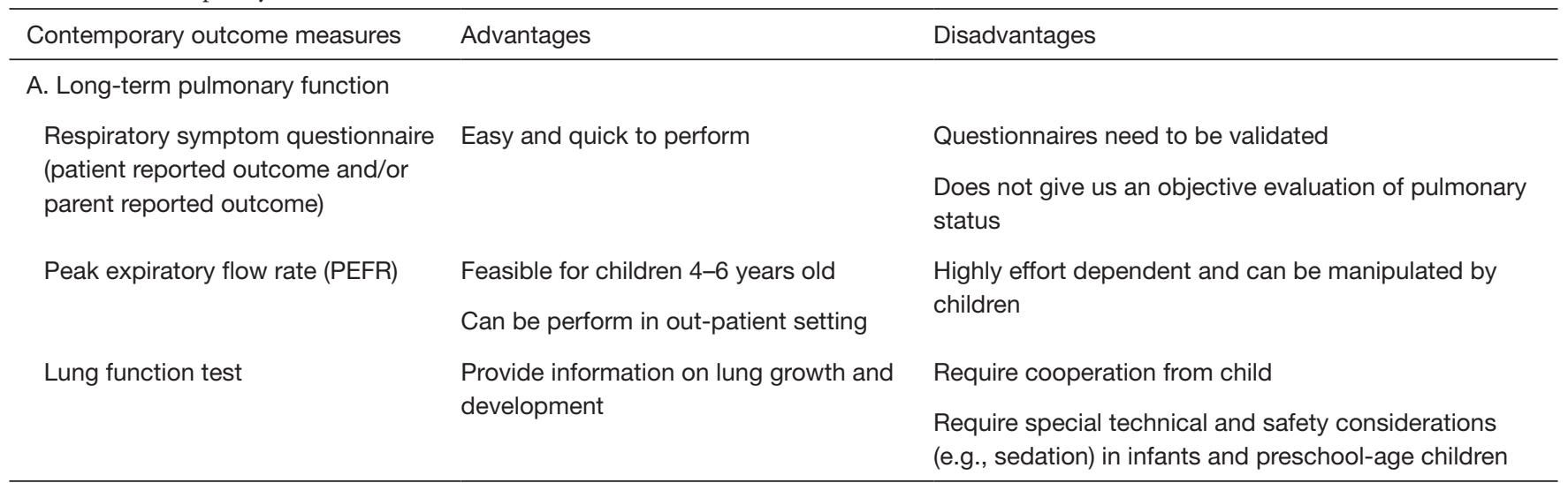

Table 2 (continued) 
Table 2 (continued)

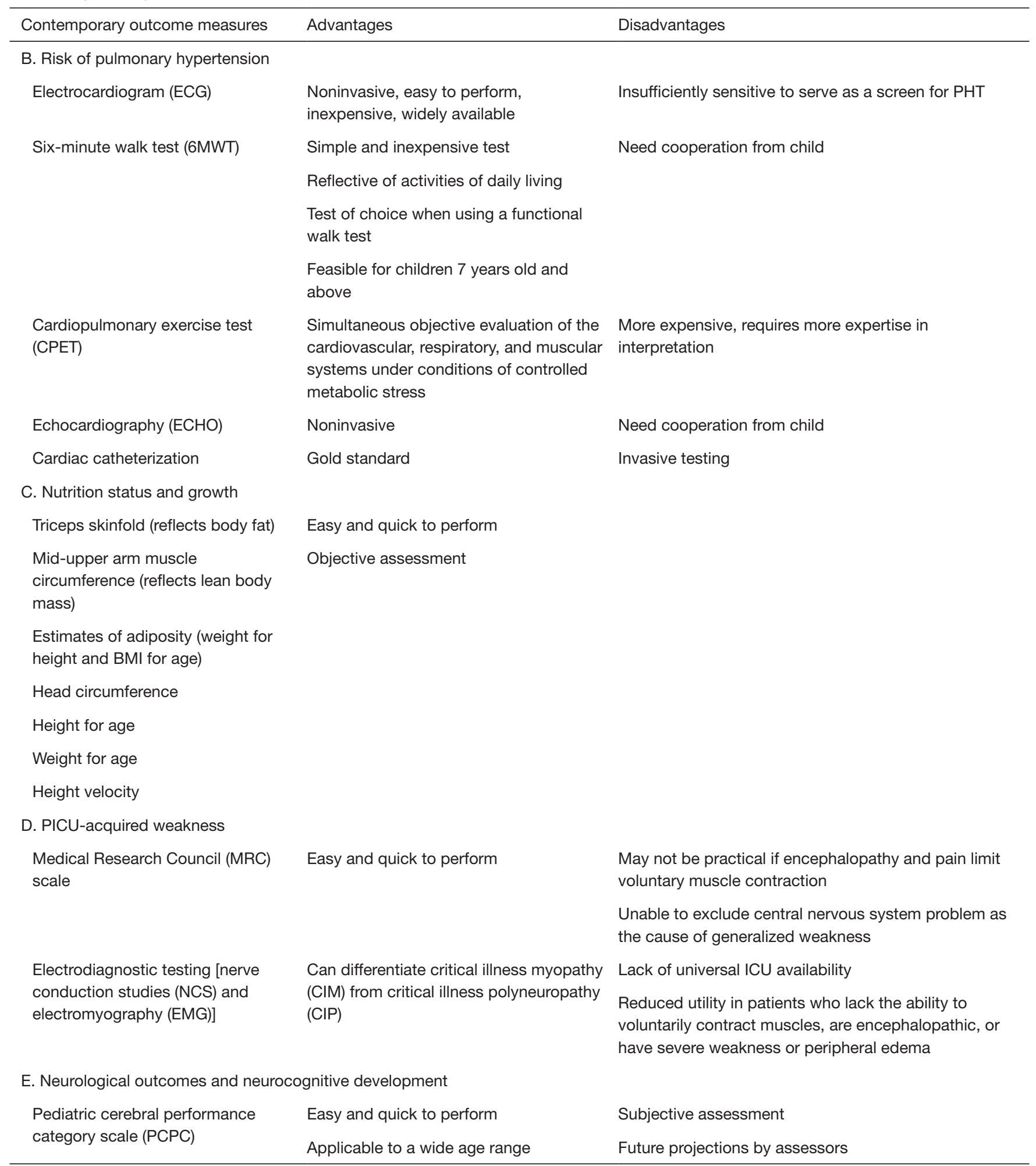

Table 2 (continued) 
Table 2 (continued)

\begin{tabular}{|c|c|c|}
\hline Contemporary outcome measures & Advantages & Disadvantages \\
\hline \multirow[t]{3}{*}{ Functional status scale (FSS) } & Easy and quick to perform & \multirow[t]{3}{*}{ It is not designed to predict long-term outcomes } \\
\hline & Applicable to a wide age range & \\
\hline & Enable large pediatric outcome studies & \\
\hline $\begin{array}{l}\text { Pediatric overall performance } \\
\text { categories (POPC) }\end{array}$ & Applicable to a wide age range & Future projections by assessors \\
\hline $\begin{array}{l}\text { Other adaptive behavior scale: } \\
\text { Adaptive Behavior Assessment }\end{array}$ & $\begin{array}{l}\text { Consider as gold standard for measuring } \\
\text { activities of adaptive skills in children }\end{array}$ & Time consuming \\
\hline $\begin{array}{l}\text { System (ABAS-III), Vineland Adaptive } \\
\text { Behavior Scales (Vineland }{ }^{\mathrm{T} M} \text {-III), } \\
\text { Bayley Scales of Infant and Toddler } \\
\text { Development (Bayley-III) }\end{array}$ & Allow long-term follow-up & \\
\hline \multicolumn{3}{|l|}{ G. Health-related quality of life (HRQOL) } \\
\hline \multirow[t]{2}{*}{ Generic versus disease-specific } & Generic gives a comprehensive overview & $\begin{array}{l}\text { Generic unable to provide a detailed or reliable } \\
\text { measurement that are specific to certain condition } \\
\text { and not sensitive to the impact of changes in clinical } \\
\text { conditions or treatment }\end{array}$ \\
\hline & $\begin{array}{l}\text { Disease-specific gives more details and } \\
\text { more sensitive to changes }\end{array}$ & $\begin{array}{l}\text { Disease-specific requires development, testing and } \\
\text { validation }\end{array}$ \\
\hline \multirow[t]{2}{*}{ Self-report versus proxy-report } & \multirow[t]{2}{*}{ Self-report-more self-reflective } & $\begin{array}{l}\text { Self-report-not applicable to young children as they } \\
\text { are unable to express themselves }\end{array}$ \\
\hline & & $\begin{array}{l}\text { Proxy-report-perceived HRQOL may not be truly } \\
\text { reflective of patients' } H R Q O L \text { and subject to bias }\end{array}$ \\
\hline \multirow{2}{*}{ H. Health-care costs } & \multirow{2}{*}{$\begin{array}{l}\text { Healthcare financial and resources } \\
\text { planning }\end{array}$} & Time-consuming \\
\hline & & Difficult to obtain \\
\hline I. Caregiver and family stress & Ensure long-term well-being of the family & Need trained personnel to perform the tests \\
\hline
\end{tabular}

quality of survivorship and these include functional status, respiratory status, growth, and quality of life of the patient and the family.

\section{Acknowledgments}

The authors thank Ms Peggy Fong Bih Yuh, head librarian of KK Women's and Children's Hospital, for assisting with the formulation of the search strategy.

\section{Footnote}

Conflicts of Interest: The authors have no conflicts of interest to declare.

Ethical Statement: The authors are accountable for all 
aspects of the work in ensuring that questions related to the accuracy or integrity of any part of the work are appropriately investigated and resolved.

\section{References}

1. Khemani RG, Smith L, Lopez-Fernandez YM, et al. Paediatric acute respiratory distress syndrome incidence and epidemiology (PARDIE): an international, observational study. Lancet Respir Med 2019;7:115-28.

2. Wong JJ, Jit M, Sultana R, et al. Mortality in Pediatric Acute Respiratory Distress Syndrome: A Systematic Review and Meta-Analysis. J Intensive Care Med 2019;34:563-71.

3. Matthay MA, McAuley DF, Ware LB. Clinical trials in acute respiratory distress syndrome: challenges and opportunities. Lancet Respir Med 2017;5:524-34.

4. Keim G, Watson RS, Thomas NJ, et al. New Morbidity and Discharge Disposition of Pediatric Acute Respiratory Distress Syndrome Survivors. Crit Care Med 2018;46:1731-8.

5. Wilcox ME, Herridge MS. Long-term outcomes in patients surviving acute respiratory distress syndrome. Semin Respir Crit Care Med 2010;31:55-65.

6. Needham DM, Davidson J, Cohen H, et al. Improving long-term outcomes after discharge from intensive care unit: report from a stakeholders' conference. Crit Care Med 2012;40:502-9.

7. Herridge MS, Tansey CM, Matté A, et al. Functional Disability 5 Years after Acute Respiratory Distress Syndrome. N Engl J Med 2011;364:1293-304.

8. Manning JC, Pinto NP, Rennick JE, et al. Conceptualizing Post Intensive Care Syndrome in Children-The PICS-p Framework. Pediatr Crit Care Med 2018;19:298-300.

9. Quasney MW, Lopez-Fernandez YM, Santschi M, et al. The outcomes of children with pediatric acute respiratory distress syndrome: proceedings from the Pediatric Acute Lung Injury Consensus Conference. Pediatr Crit Care Med 2015;16:S118-31.

10. Hayes JA, Black NA, Jenkinson C, et al. Outcome measures for adult critical care: a systematic review. Health Technol Assess 2000;4:1-111.

11. Yehya N, Keim G, Thomas NJ. Subtypes of pediatric acute respiratory distress syndrome have different predictors of mortality. Intensive Care Med 2018;44:1230-9.

12. Gan CS, Wong JJ, Samransamruajkit R, et al. Differences Between Pulmonary and Extrapulmonary Pediatric Acute Respiratory Distress Syndrome: A Multicenter Analysis.
Pediatr Crit Care Med 2018;19:e504-13.

13. Rubenfeld GD. Who cares about preventing acute respiratory distress syndrome? Am J Respir Crit Care Med 2015;191:255-60.

14. Wang CY, Calfee CS, Paul DW, et al. One-year mortality and predictors of death among hospital survivors of acute respiratory distress syndrome. Intensive Care Med 2014;40:388-96.

15. Yehya N, Servaes S, Thomas NJ. Characterizing degree of lung injury in pediatric acute respiratory distress syndrome. Crit Care Med 2015;43:937-46.

16. Yehya N, Thomas NJ. Relevant Outcomes in Pediatric Acute Respiratory Distress Syndrome Studies. Front Pediatr 2016;4:51.

17. Curley MA, Hibberd PL, Fineman LD, et al. Effect of prone positioning on clinical outcomes in children with acute lung injury: a randomized controlled trial. Jama 2005;294:229-37.

18. Bronicki RA, Fortenberry J, Schreiber M, et al. Multicenter randomized controlled trial of inhaled nitric oxide for pediatric acute respiratory distress syndrome. J Pediatr 2015;166:365-9.e1.

19. Drago BB, Kimura D, Rovnaghi CR, et al. Doubleblind, placebo-controlled pilot randomized trial of methylprednisolone infusion in pediatric acute respiratory distress syndrome. Pediatr Crit Care Med 2015;16:e74-81.

20. Gajic O, Dabbagh O, Park PK, et al. Early identification of patients at risk of acute lung injury: evaluation of lung injury prediction score in a multicenter cohort study. Am J Respir Crit Care Med 2011;183:462-70.

21. Ranieri VM, Rubenfeld GD, Thompson BT, et al. Acute respiratory distress syndrome: the Berlin Definition. JAMA 2012;307:2526-33.

22. Curley MA, Wypij D, Watson RS, et al. Protocolized sedation vs. usual care in pediatric patients mechanically ventilated for acute respiratory failure: a randomized clinical trial. JAMA 2015;313:379-89.

23. Brasel KJ, Lim HJ, Nirula R, et al. Length of stay: an appropriate quality measure? Arch Surg 2007;142:461-5; discussion 465-6.

24. Chakdour S, Vaidya PC, Angurana SK, et al. Pulmonary Functions in Children Ventilated for Acute Hypoxemic Respiratory Failure. Pediatr Crit Care Med 2018;19:e464-71.

25. Ward SL, Turpin A, Spicer AC, et al. Long-Term Pulmonary Function and Quality of Life in Children After Acute Respiratory Distress Syndrome: A Feasibility Investigation. Pediatr Crit Care Med 2017;18:e48-55.

26. Ben-Abraham R, Weinbroum AA, Roizin H, et al. Long- 
term assessment of pulmonary function tests in pediatric survivors of acute respiratory distress syndrome. Med Sci Monit 2002;8:CR153-7.

27. Leeman M. Pulmonary hypertension in acute respiratory distress syndrome. Monaldi Arch Chest Dis 1999;54:146-9.

28. Himebauch AS, Yehya N, Wang Y, et al. Early Right Ventricular Systolic Dysfunction and Pulmonary Hypertension Are Associated With Worse Outcomes in Pediatric Acute Respiratory Distress Syndrome. Crit Care Med 2018;46:e1055-62.

29. Vieillard-Baron A, Schmitt JM, Augarde R, et al. Acute cor pulmonale in acute respiratory distress syndrome submitted to protective ventilation: incidence, clinical implications, and prognosis. Crit Care Med 2001;29:1551-5.

30. Calcaianu G, Calcaianu M, Gschwend A, et al. Hemodynamic profile of pulmonary hypertension $(\mathrm{PH})$ in ARDS. Pulm Circ 2018;8:2045893217753415.

31. Ryan D, Frohlich S, McLoughlin P. Pulmonary vascular dysfunction in ARDS. Ann Intensive Care 2014;4:28.

32. Price LC, McAuley DF, Marino PS, et al. Pathophysiology of pulmonary hypertension in acute lung injury. Am J Physiol Lung Cell Mol Physiol 2012;302:L803-15.

33. Vieillard-Baron A, Price LC, Matthay MA. Acute cor pulmonale in ARDS. Intensive Care Med 2013;39:1836-8.

34. Tran DL, Lau EMT, Celermajer DS, et al. Pathophysiology of exercise intolerance in pulmonary arterial hypertension. Respirology 2018;23:148-59.

35. Solway S, Brooks D, Lacasse Y, et al. A Qualitative Systematic Overview of the Measurement Properties of Functional Walk Tests Used in the Cardiorespiratory Domain. Chest 2001;119:256-70.

36. Dimopoulos K, Diller GP, Piepoli MF, et al. Exercise intolerance in adults with congenital heart disease. Cardiol Clin 2006;24:641-60, vii.

37. Lammers AE, Diller GP, Odendaal D, et al. Comparison of 6-min walk test distance and cardiopulmonary exercise test performance in children with pulmonary hypertension. Arch Dis Child 2011;96:141-7.

38. Sawada H, Mitani Y, Nakayama T, et al. Detection of Pediatric Pulmonary Arterial Hypertension by School Electrocardiography Mass Screening. Am J Respir Crit Care Med 2019;199:1397-406.

39. Nakatsuji A, Miyauchi Y, Iwasaki YK, et al. Detection and Evaluation of Pulmonary Hypertension by a Synthesized Right-Sided Chest Electrocardiogram. J Nippon Med Sch 2015;82:136-45
40. Whitman IR, Patel VV, Soliman EZ, et al. Validity of the surface electrocardiogram criteria for right ventricular hypertrophy: the MESA-RV Study (Multi-Ethnic Study of Atherosclerosis-Right Ventricle). J Am Coll Cardiol 2014;63:672-81.

41. Lehtonen J, Sutinen S, Ikaheimo M, et al. Electrocardiographic criteria for the diagnosis of right ventricular hypertrophy verified at autopsy. Chest 1988;93:839-42.

42. Apitz C, Hansmann G, Schranz D. Hemodynamic assessment and acute pulmonary vasoreactivity testing in the evaluation of children with pulmonary vascular disease. Expert consensus statement on the diagnosis and treatment of paediatric pulmonary hypertension. The European Paediatric Pulmonary Vascular Disease Network, endorsed by ISHLT and DGPK. Heart 2016;102 Suppl 2:ii23-9.

43. Arcasoy SM, Christie JD, Ferrari VA, et al. Echocardiographic assessment of pulmonary hypertension in patients with advanced lung disease. Am J Respir Crit Care Med 2003;167:735-40.

44. Mehta NM, McAleer D, Hamilton S, et al. Challenges to optimal enteral nutrition in a multidisciplinary pediatric intensive care unit. JPEN J Parenter Enteral Nutr 2010;34:38-45.

45. Wong JJ, Han WM, Sultana R, et al. Nutrition Delivery Affects Outcomes in Pediatric Acute Respiratory Distress Syndrome. JPEN J Parenter Enteral Nutr 2017;41:1007-13.

46. Mehta NM, Bechard LJ, Cahill N, et al. Nutritional practices and their relationship to clinical outcomes in critically ill children--an international multicenter cohort study*. Crit Care Med 2012;40:2204-11.

47. Mehta NM, Bechard LJ, Zurakowski D, et al. Adequate enteral protein intake is inversely associated with 60-d mortality in critically ill children: a multicenter, prospective, cohort study. Am J Clin Nutr 2015;102:199-206.

48. Mikhailov TA, Kuhn EM, Manzi J, et al. Early enteral nutrition is associated with lower mortality in critically ill children. JPEN J Parenter Enteral Nutr 2014;38:459-66.

49. Wilson B, Typpo K. Nutrition: A Primary Therapy in Pediatric Acute Respiratory Distress Syndrome. Front Pediatr 2016;4:108.

50. Puthucheary ZA, Rawal J, McPhail M, et al. Acute skeletal muscle wasting in critical illness. JAMA 2013;310:1591-600.

51. Rennie MJ. Muscle protein turnover and the wasting due to injury and disease. Br Med Bull 1985;41:257-64. 
52. Ward SL, Gildengorin V, Valentine SL, et al. Impact of Weight Extremes on Clinical Outcomes in Pediatric Acute Respiratory Distress Syndrome. Crit Care Med 2016;44:2052-9.

53. Meduri GU, Headley AS, Golden E, et al. Effect of prolonged methylprednisolone therapy in unresolving acute respiratory distress syndrome: a randomized controlled trial. JAMA 1998;280:159-65.

54. Meduri GU, Bridges L, Shih MC, et al. Prolonged glucocorticoid treatment is associated with improved ARDS outcomes: analysis of individual patients' data from four randomized trials and trial-level meta-analysis of the updated literature. Intensive Care Med 2016;42:829-40.

55. Abrantes MA, Valencia AM, Bany-Mohammed F, et al. Dose response effects of postnatal hydrocortisone on growth and growth factors in the neonatal rat. Steroids 2018;140:1-10.

56. Tijsseling D, Ter Wolbeek M, Derks JB, et al. Neonatal corticosteroid therapy affects growth patterns in early infancy. PLoS One 2018;13:e0192162.

57. Hermans G, De Jonghe B, Bruyninckx F, et al. Clinical review: Critical illness polyneuropathy and myopathy. Crit Care 2008;12:238.

58. Field-Ridley A, Dharmar M, Steinhorn D, et al. ICUAcquired Weakness Is Associated With Differences in Clinical Outcomes in Critically Ill Children. Pediatr Crit Care Med 2016;17:53-7.

59. Baud O, Trousson C, Biran V, et al. Association Between Early Low-Dose Hydrocortisone Therapy in Extremely Preterm Neonates and Neurodevelopmental Outcomes at 2 Years of Age. JAMA 2017;317:1329-37.

60. Malaeb SN, Stonestreet BS. Steroids and injury to the developing brain: net harm or net benefit? Clin Perinatol 2014;41:191-208.

61. Pollack MM, Glass P, Dean JM, et al. The functional status score: A New Pediatric Outcome Measure. Pediatrics

Cite this article as: Lee SW, Loh SW, Ong C, Lee JH. Pertinent clinical outcomes in pediatric survivors of pediatric acute respiratory distress syndrome (PARDS): a narrative review. Ann Transl Med 2019;7(19):513. doi: 10.21037/atm.2019.09.32 2009;124:e18-28.

62. Pollack MM, Holubkov R, Funai T, et al. Simultaneous Prediction of New Morbidity, Mortality, and Survival Without New Morbidity From Pediatric Intensive Care: A New Paradigm for Outcomes Assessment. Crit Care Med 2015;43:1699-709.

63. Fiser DH. Assessing the outcome of pediatric intensive care. J Pediatr 1992;121:68-74.

64. Haverman L, Limperg PF, Young NL, et al. Paediatric health-related quality of life: what is it and why should we measure it? Arch Dis Child 2017;102:393-400.

65. Grootenhuis MA, Koopman HM, Verrips EGH, et al. Health-related quality of life problems of children aged 8-11 years with a chronic disease. Developmental Neurorehabilitation 2007;10:27-33.

66. Angus DC, Musthafa AA, Clermont G, et al. Qualityadjusted survival in the first year after the acute respiratory distress syndrome. Am J Respir Crit Care Med 2001;163:1389-94.

67. Clark ME, Cummings BM, Kuhlthau K, et al. Impact of Pediatric Intensive Care Unit Admission on Family Financial Status and Productivity: A Pilot Study. J Intensive Care Med 2017:885066617723278.

68. Ho K, Wang X, Chen L. Reasons for parental withdrawal of care in a pediatric intensive care unit in China. PLoS One 2018;13:e0199419.

69. Gupta P, Rettiganti M. Relationship of Hospital Costs With Mortality in Pediatric Critical Care: A Multi-Institutional Analysis. Pediatr Crit Care Med 2017;18:541-9.

70. Board R, Ryan-Wenger N. Long-term effects of pediatric intensive care unit hospitalization on families with young children. Heart Lung 2002;31:53-66.

71. Stremler R, Haddad S, Pullenayegum E, et al. Psychological Outcomes in Parents of Critically Ill Hospitalized Children. J Pediatr Nurs 2017;34:36-43. 